\title{
Guest Editorial: Wireless multimedia transmission technology and application
}

\author{
Gabriel-Miro Muntean · Pascal Frossard • \\ Haohong Wang $\cdot$ Yan Zhang $\cdot$ Liang Zhou
}

Published online: 11 November 2010

(C) Springer-Verlag 2010

The rapid expansion of wireless services from voice communication to high performance multimedia communication in recent years is an indication that significant value is placed on portable multimedia applications as key innovations for both academia and industry. The emerging wireless multimedia transmission techniques can be used for various pervasive and ubiquitous applications such as health care, environmental monitoring, security and surveillance. There are a number of important challenges that arise when time-constrained multimedia streams are transmitted in these new wireless systems.

This exciting special issue has received 16 submissions from an open call for papers that cover all topics of wireless multimedia technologies and applications. Due to page budget and timing constraints, many good submissions have been turned away, and seven papers (including one invited paper) have finally been selected after a careful and highly competitive review process. These papers cover diversified issues, including wireless multimedia network and

G.-M. Muntean

School of Electronic Engineering, Dublin City University,

Dublin, Ireland

P. Frossard

Signal Processing Laboratory (LTS4), Ecole Polytechnique

Fédérale de Lausanne (EPFL), Lausanne, Switzerland

H. Wang

Cisco Systems, San Jose, USA

Y. Zhang

Simula Research Laboratory, Lysaker, Norway

L. Zhou ( $ه)$

Technical University of Munich, Munich, Germany

e-mail: liang.zhou@ieee.org application, transmission technology, information processing, and multimedia services over wireless networks.

The first paper of this special issue is an invited paper on wireless multimedia network and application. The paper "Wireless MediaNets: Application Driven Next Generation Wireless IP Networks," by J.-N. Hwang addresses the QoS challenges specifically encountered in video over heterogeneous wireless broadband networks, and addresses several application-driven wireless MediaNet solutions based on effective cross-layer integration of application and MAC/PHY layers. More specifically, the congestion control for achieving airtime fairness of video streaming to maximize the link adaptation performance of $\mathrm{Wi}-\mathrm{Fi}$, the minimum latency event-driven data exchange for distributed wireless ad hoc sensor networks, and the opportunistic multicast of scalable video live streaming over WiMAX.

The second set of two papers describes the emerging wireless multimedia transmission technology. The first paper, "Real-time Video Streaming over Multipath in Multi-hop Wireless Networks", by X. Guo, J. Liu and S. Lian, proposes a novel cross-layer design with a smart traffic split scheme, namely, multiple path retransmission. It differentiates the original data packets and the retransmitted packets, and works with a novel QoS-aware multipath routing protocol, QAOMDV, to distribute them separately. It does not suffer from the round trip time underestimation and extra packet reordering, which ensures stable throughput improvement over single path routing. The second paper, "Design and Integration of the OpenCore based Mobile TV framework for DVB-H/ T Wireless Network", by C.-F. Lai, Y.-M. Huang, J.-L. Chen, W. Ji and M. Chen, incorporates the function of mobile TV into the OpenCore framework, in order to support both formats of TV signals, i.e. DVB-H and DVB-T. The incorporated function, DVB-H/T, has different characteristics, so that 
users can select TV signals according to their receiving environments and fulfill their needs in TV program selection.

The next set of two papers deals with multimedia information processing in wireless networks. The paper "M-MUSICS: An Intelligent Mobile Music Retrieval System," by S. Rho, E. Hwang, and J.H. Park, proposes a new voice query transcription scheme. It considers the following features: note onset detection using dynamic threshold methods, fundamental frequency acquisition of each frame, and frequency realignment using K-means. In addition, a popularity-adaptive indexing structure called Frequently Accessed Index is used for indexing purposes. Another paper, "Adaptive Hybrid Error Correction Model for Video Streaming over Wireless Networks," by M.-F. Tsai, T.-C. Huang, C.-H. Ke, C.-K. Shieh, and W.-S. Hwang, proposes an Adaptive Hybrid Error Correction Model (AHECM) to improve the Hybrid ARQ mechanism. The AHECM can limit the packet retransmission delay to the most tolerable end-to-end delay. Besides, the AHECM can find the appropriate forward error correction parameter to avoid network congestion and reduce the number of forward error correction redundant packets by predicting the effective packet loss rate.

The final set of two papers addresses multimedia services over wireless networks. The paper "The Potential Cyberterrorism via a Multimedia Smart Phone based on a Web 2.0 Application via ubiquitous Wi-Fi Access Points and the Corresponding Digital Forensics," by H.-C. Chu, D.-J. Deng and H.-C. Chao, provides a hypothetical case review concerning the digital forensics of a potential cyber terrorist attack that was triggered by a mobile multimedia smart phone utilizing a popular web 2.0 application program via ubiquitous $\mathrm{Wi}-\mathrm{Fi}$ access points. The paper "IPTV Service based on a Content-Zapping Paradigm," by J. Rodrigues, A. Nogueira, P. Salvador, J.J.P.C. Rodrigues, describes a general functionality of the service and presents a detailed architecture of the IPTV server, the key component of the service infrastructure. These contextawareness mechanisms applied to mobile devices with wireless network $(802.11 \mathrm{~b} / \mathrm{g} / \mathrm{n}$, WiMAX, GSM, UMTS, etc.) capabilities allow a better user experience and more accurate multimedia recommendations, due to the deep knowledge of the user device, network and environment.

The guest editorial team would like to thank all authors for submitting their quality work to this special issue, and to the numerous reviewers whose hard work and expert contributions are certainly the cornerstone in the quality of this successful special issue. Finally, special thanks go to Prof. Thomas Plagemann, Editor-in-Chief for approving and making this issue possible, and to Prof. Cormac
J. Sreenan for his priceless guidance along the whole process of this special issue.

\section{Author Biographies}

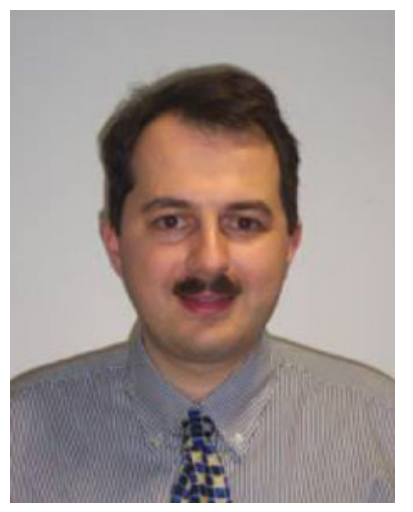

Gabriel-Miro Muntean is a Lecturer with the School of Electronic Engineering and coDirector of the Performance Engineering Laboratory at Dublin City University, Ireland. He obtained his Ph.D. degree from Dublin City University, Ireland for research in the area of quality-oriented adaptive multimedia streaming in 2003. He was awarded the B.Eng. and M.Sc. degrees in Software Engineering from the Computer Science Department, "Politehnica" University of Timisoara, Romania in 1996 and 1997, respectively. Dr. Muntean's research interests include quality and performance-related issues of adaptive multimedia streaming, and personalized eLearning over wired and wireless networks and with various devices. Dr. Muntean has published over 100 papers in toplevel international conferences and journals and has authored a book and five book chapters and edited two books. Dr. Muntean is Associate Editor with the IEEE Transactions on Broadcasting and reviewer for important international journals, conferences and funding agencies. He is member of IEEE, IEEE Broadcast Society and Research Institute for Networks and Communications Engineering (RINCE) Ireland.

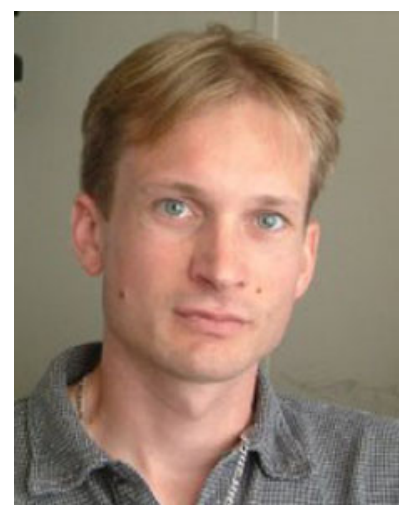

Pascal Frossard received the M.S. and Ph.D. degrees, both in electrical engineering, from the Swiss Federal Institute of Technology (EPFL), Lausanne, Switzerland, in 1997 and 2000, respectively. Between 2001 and 2003, he was a member of the research staff at the IBM T.J. Watson Research Center, Yorktown Heights, NY, where he worked on media coding and streaming technologies. Since 2003, he has been an assistant professor at EPFL, where he heads the Signal Processing Laboratory (LTS4). His research interests include image representation and coding, visual information analysis, distributed image processing and communications, and media streaming systems. Dr. Frossard has been the General Chair of IEEE ICME 2002 and Packet Video 2007. He has been the Technical Program Chair of EUSIPCO 2008, and a member of the organizing or technical program committees of numerous conferences. He has been an Associate Editor of the IEEE Transactions on Multimedia (2004-2010), the IEEE Transactions on Image Processing (2010-) and the IEEE Transactions on Circuits and Systems for Video Technology (2006-). He is an elected member of the IEEE Image and Multidimensional Signal Processing Technical Committee (2007-), the IEEE Visual Signal Processing and Communications Technical Committee 
(2006-), and the IEEE Multimedia Systems and Applications Technical Committee (2005-). He has served as Vice-Chair of the IEEE Multimedia Communications Technical Committee (2004-2006) and as a member of the IEEE Multimedia Signal Processing Technical Committee (2004-2007).

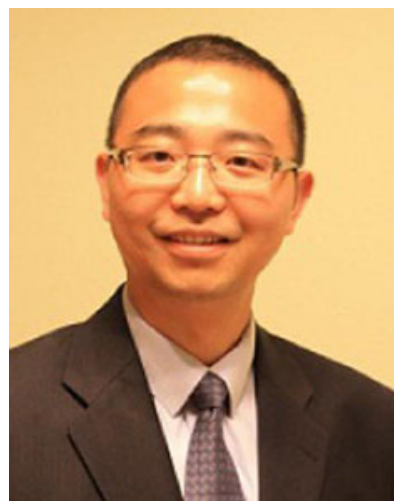

Haohong Wang received the B.S. degree in computer science and the M. Eng. degree in computer applications both from Nanjing University, China, the M.S. degree in computer science from University of New Mexico, and the Ph.D. degree in Electrical and computer engineering from Northwestern University, Evanston, USA. He is currently a Principal Software Architect and Senior Technology Manager at TCL-Thomson Electronics at Santa Clara, CA, managing a US/Singapore/HK/China-based world-class R\&D team for visual processing. Prior to joining this world largest TV maker, he held various technical and management positions at AT\&T, Catapult Communications, Qualcomm and Marvell. Dr. Wang's research involves the areas of multimedia communications, 3D graphics, and video analysis and processing. He has published more than 40 articles in peer-reviewed journals and International conferences. He is the inventor of more than 40 US patents and pending applications. He is the co-author of 4G Wireless Video Communications (Wiley, 2009), and Computer Graphics (1997).

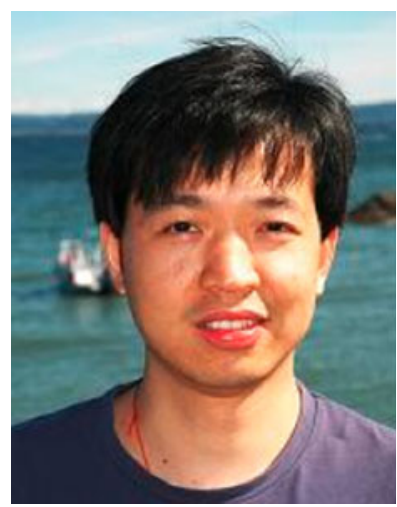

Yan Zhang received his Ph.D. degree from Nanyang Technological University, Singapore. From August 2006, he is working with Simula Research Laboratory, Norway. $\mathrm{He}$ is currently heading the "Wireless Networks" research group at Simula Research Laboratory, Norway. He is an Associate Professor (part-time) at the University of Oslo, Norway. He is a regional editor, associate editor, on the editorial board, or guest editor of a number of international journals. He serves as organizing committee chairs for many international conferences. His research interests include resource, mobility, spectrum, energy, and data management in wireless communications and networking.

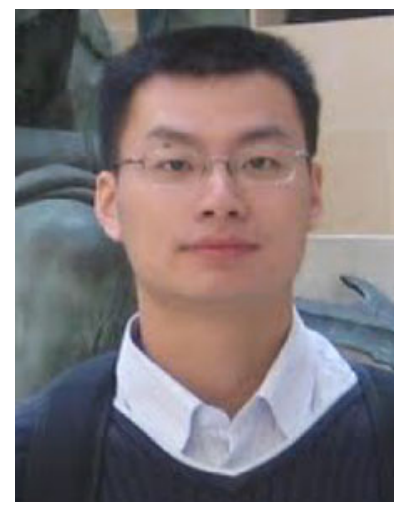

Liang Zhou received his Ph.D. degree major at Electronic Engineering both from Ecole Normale Superieure (E.N.S.), Cachan, France and Shanghai Jiao Tong University, Shanghai, China in March 2009. From 2009 to 2010 , he is a postdoctoral researcher in ENSTAParisTech, Paris, France. Since October 2010, he is an Alexander von Humboldt Research Fellow in Munich University of Technology, Munich, Germany. His research interests are in the area of network-based multimedia communications and computing, in particular, resource allocation, system scheduling, cross-layer design, multimedia security, multimedia signal processing. He is a member of IEEE, IEEE ComSoc, and IEEE ComSoc Multimedia Communications Technical Committee. 